IC Vol. $5 N^{\circ}$ 2: pp. 217-232, 2014

\title{
PROGRAMA DE SEGURIDAD INDUSTRIAL MEJORA LA PRODUCTIVIDAD EN EMPRESAS DE CURTIEMBRE DE TRUJILLO, 2013*
}

\author{
INDUSTRIAL SAFETY PROGRAMS IMPROVE PRODUCTIVITY \\ OF TANNING ENTERPRISES IN TRUJILLO, 2013
}

\author{
Yuri Gagarin Gonzales Renteria ${ }^{1}$
}

\begin{abstract}
RESUMEN
El propósito de la investigación es determinar la influencia de la aplicación de un programa de seguridad industrial en la productividad de la empresa curtiembre Chimú Murguía Hermanos SAC. de la ciudad de Trujillo, 2013. El diseño de la investigación ha sido descriptivocorrelacional. El problema de investigación fue ¿En qué medida influye la aplicación de un Programa de seguridad industrial en la productividad de la empresa "Curtiembre Chimú Murguía HNOS S.A.C” de la ciudad de Trujillo, año 2013? La hipótesis fue: la aplicación de un Programa de seguridad industrial influye significativamente en la productividad de la empresa "Curtiembre Chimú Murguía HNOS S.A.C" de la ciudad de Trujillo, año 2013. En el material de estudio se ha tomado como muestra a la curtiembre Chimú Murguía hermanos de la ciudad de Trujillo. La aplicación de un sistema de seguridad permite evaluar posibles riesgos y minimizarlos, logrando así que el personal no sufra accidentes ni enfermedades ocupacionales que pudieran traer como consecuencia la ausencia del mismo a su puesto, no solo de labores, sino consecuencias para el propio trabajador; busca prevenir riesgos y crear conciencia en seguridad en el personal, a fin de darse cuenta de que los accidentes generan pérdida. Se concluye que la aplicación de un programa de seguridad industrial es de vital importancia, ya que con ello se establecerá las condiciones óptimas de funcionamiento para reducir sustancialmente los parámetros estadísticos de accidentes laborales y siniestros; así mismo se reducirá el nivel de improductividad.
\end{abstract}

PALABRAS CLAVE: Programa de seguridad industrial, productividad, curtiembre.

* Recibido: 21 agosto 2014; aprobado: 21 noviembre 2014.

1 Doctor en Contabilidad y Finanzas, Magister en Administración, asesor de empresas, docente contratado de la carrera profesional de Contabilidad - ULADECH Católica - Sede Central. E-mail: yisguerul@hotmail.com,www.yurigonzales.com 


\section{ABSTRACT}

This cross sectional study aimed to determine the influence of the implementation of a industrial safety program in the tannery business productivity Chimu Murguía Brothers SAC. Trujillo City. 2013. It was a descriptive and correlational research that took place in the own enterprise. It is concluded that the implementation of a program of industrial safety is of vital importance, since this optimal operating conditions reduces the statistical parameters of occupational accidents and casualties.

KEY WORDS: Tannery, productivity, industrial security program.

\section{INTRODUCCIÓN}

El concepto de lo seguro ha acompañado a la evolución de las sociedades desde su inicio en los albores de la historia. La seguridad se relaciona necesaria e íntimamente con la propia supervivencia de la especie humana, de los individuos y las personas que la componen, dotándola de pervivencia y de un profundo sentido vital como condición necesaria para la realización de sus actividades.

Es fundamental aseverar que el desarrollo económico, infraestructural e industrial de un país debe estar inmerso implícitamente con su productividad y los efectos de esta en el desarrollo integral. Este trabajo tiene como objetivo entregar las pautas apropiadas para resaltar la importancia de las condiciones de seguridad necesarias para áreas de trabajo (Ceballos 2010).

El recurso humano representa el factor clave en el mejoramiento de la productividad, por lo que es de suma importancia el estudio de las condiciones de seguridad en las instalaciones de la empresa en la que labora. La función de la seguridad dentro de la organización ha establecido dos frentes íntimamente ligados: por un lado, se establece la necesidad de la conservación física de los diferentes bienes con los que cuenta la empresa para operar tanto dentro como fuera de sus instalaciones; por otro lado, la conservación física y la salud de la fuerza laboral.

Incrementar la productividad de una empresa a través de una correcta implementación de un programa de seguridad industrial derivado del presente trabajo, implica que esta se debe realizar con eficacia y estimular a quienes intervienen en él, para su adecuada ejecución.

Con el presente contenido podremos adentrarnos en la complejidad del concepto de la seguridad y adquirir o aumentar los conocimientos que se deben considerar para lograr condiciones de trabajo adecuadas para las operaciones de cualquier empresa en la actualidad; los mismos que son necesarios para la solu- 
ción de problemas relacionados con la seguridad. Esto se buscará al exponer una clara idea de lo que es la seguridad industrial y el papel de las auditorias de seguridad; de la misma manera, los diferentes factores a considerar dentro del puesto de trabajo para la mejora del ambiente laboral, la investigación y eliminación de condiciones, que de no considerarse, podrían traducirse en accidentes contraproducentes en el desempeño de las actividades productivas (Harrinson 2011).

Finalmente, se exponen los resultados de un estudio de las condiciones de seguridad en una empresa del sector de la industria del cuero de calzado, que en concordancia a lo que sugieren los diversos factores a considerarse en el campo de la seguridad, de manera que el presente trabajo sirva de base para una futura creación e implementación de un programa de seguridad en dicha empresa; buscando de esa manera, la evaluación y establecimiento de condiciones apropiadas en los ambientes de trabajo, para que su aplicación en la empresa objeto de estudio refleje efectos en pro de la productividad en la misma.

La auditoría de seguridad es la evaluación objetiva del sistema de administración de la seguridad de la empresa, la cual permite:

a) Realizar revisiones sistemáticas de los procesos y procedimientos para evaluar el desempeño del propio sistema;

b) Ayudar a los empleados a mantener unos con otros los niveles deseados de calidad y seguridad, así como altos estándares de autodisciplina.

c) Crear cimientos documentados para el mejoramiento continuo del sistema de seguridad. La realización de auditorías de seguridad dentro de la empresa representa una gran ayuda a la administración, ya que son verificaciones rutinarias y sistemáticas para vigilar que los procedimientos escritos sigan y sean seguros. No solo contribuyen con el fortalecimiento de la administración, sino que dan autoridad a los empleados para que se ayuden mutuamente en el mejoramiento de sus sistemas y desempeños.

La revisión sobre una base regular de los procesos evita que se deslicen errores en los productos y los servicios, al reducirse al máximo las fallas en el cumplimiento de las especificaciones y los problemas de servicio, por lo que la administración obtiene pruebas objetivas del desempeño del sistema y de las oportunidades de mejoramiento. Con la documentación y solución de fallas en el cumplimiento de las especificaciones se previene la aparición de problemas similares (acciones preventivas).

La utilidad de las auditorías en la empresa dentro del concepto de seguri- 
dad, proviene de la planeación que estas establecen para la revisión de todas las áreas del sistema de administración de seguridad de las operaciones. Las auditorías internas sirven como base para cambiar y mejorar los procedimientos y procesos, así como de base de datos para que la administración elabore planes estratégicos y aplique acciones preventivas.

Por lo general un equipo de dos o más personas que trabajan fuera del área auditada llevan a cabo auditorías, las cuales tienen una agenda específica y se concentran en un procedimiento o proceso específico, no en un apersona.

El formato general de una inspección considerara:

- Selección de procedimiento o proceso a auditar.

- Determinación de quien forma parte del equipo auditor.

- Notificación del tema a la persona responsable del área que se auditará y establecimiento del momento oportuno para ambas partes.

- Leer con mucho cuidado el procedimiento operativo o el procedimiento del proceso y realizar una lista de verificación.

- Llevar a cabo la auditoría con una persona del área como guía.

- Cuando se concluya, comentar los hallazgos preliminares con la persona responsable.

- Realizar un informe de auditoría y uno de acciones correctivas, si es necesario.

- Seguir los procedimientos establecidos para distribución, respuesta, seguimiento y cierre de las acciones correctivas.

Una auditoría bien ejecutada es benéfica para todos los que toman parte, ya que si se realiza en forma deficiente provoca resentimiento y enfrentamientos. Se considera que los elementos más importantes de la auditoría son cómo considera el auditor su función y cómo se transmite esa consideración a los auditados.

El auditor debe estar convencido de que el servicio que presta es valioso, ya que tiene que participar con la mente abierta respecto de los hallazgos que encontrará durante la auditoría. Es muy importante que sea paciente y realice la auditoría siguiendo todos los pasos lógicos hasta su finalización antes de sacar conclusiones.

La auditoría más benéfica en cualquier empresa es en la que todos los empleados tengan oportunidad de ser integrantes de un equipo de inspección y que todos los empleados sean auditados. Al estar en ambos extremos de una auditoría, el círculo se cierra y hay equilibrio. A pesar de ello, hay personas que no deben ser auditores, porque su personalidad y antecedentes no les permiten 
realizar este trabajo; por lo tanto, no todos tendrán la oportunidad de desempeñar en esa actividad (Coopers \& Lybrand 2010).

\section{PROBLEMA}

¿En qué medida influye la aplicación de un Programa de seguridad industrial en la productividad de la Empresa "Curtiembre Chimú Murguía HNOS S.A.C” de la ciudad de Trujillo, año 2013?

\section{HIPÓTESIS}

La aplicación de un Programa de seguridad industrial influye significativamente en la productividad de la empresa: "Curtiembre Chimú Murguía HNOS S.A.C” de la ciudad de Trujillo, año 2013.

\section{OBJETIVO GENERAL}

Determinar la influencia de la aplicación de un Programa de seguridad industrial en la productividad de la Empresa Curtiembre Chimú Murguía Hnos. S.A.C de la ciudad de Trujillo, año 2013.

\section{MATERIALES Y MÉTODOS}

\section{MATERIAL DE ESTUDIO}

La población está constituida por los distintos sectores de la Empresa "Curtiembre Chimú Murguía HNOS S.A.C” de la ciudad de Trujillo. La muestra está constituida por el sector operativo-industrial de la Empresa "Curtiembre Chimú Murguía HNOS S.A.C” de la ciudad de Trujillo.

\section{MÉTODOS Y TÉCNICAS}

\section{MÉTODOS}

Hermenéutica. Aplicada a la interpretación de textos, análisis de los datos obtenidos mediante las encuestas y entrevistas; deductivo, que nos permitió, a partir de la observación y la interpretación de los datos obtenidos, identificar la influencia en la productividad de la empresa en estudio.

\section{TÉCNICAS}

En el desarrollo de la investigación se utilizaron las técnicas de investigación siguientes: 
Recolección de datos. A través de textos, documentos y materiales relacionados con el tema.

Cuestionario. Parte del trabajo de campo consistirá en la aplicación de encuestas a la población seleccionada como muestra.

Internet. Para obtener información sobre el tema.

\section{RESULTADOS}

\section{Seguridad Industrial: Guía de auditoria}

Condiciones actuales.

Departamento: Administración.

Fecha de inspección: 5 de agosto del 2013.

Auditor. SLCA

\section{PASILLOS E INSTALACIONES}

¿Los atraviesan mangueras o cables eléctricos?

¿Se encuentran plenamente identificados?

¿Se cumplen normas de orden de limpieza de forma que no dificulte la circulación en los pasillos?

¿Se cuenta con la debida señalización cromática en las diferentes áreas de trabajo?

¿Son adecuadas las condiciones de iluminación en el área?

¿Son adecuadas las condiciones de temperatura y ventilación en el área?

¿Existe mucho ruido en el área de trabajo?

¿Existe mucha vibración en el área de trabajo?

¿Se encuentra plenamente identificado el equipo de primeros auxilios en las áreas?

¿Se sobrecargan los tomacorrientes?

¿Se encuentran en buenas condiciones las paredes y techos de las instalaciones?

¿El piso instalado en cada área es el adecuado según las tareas que se realizan?

¿Se encuentra en buenas condiciones el sistema de cableado eléctrico de la empresa?

¿Se localiza algún material combustible cerca de la fuente de energía?

Ponderación del área:

SI NO POND.

3

4

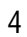

$\begin{array}{lll} & \checkmark & 2 \\ \checkmark & & 5 \\ \checkmark & & 4 \\ & \checkmark & 4 \\ & & 3\end{array}$

3

1

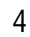

4

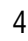




\begin{tabular}{lccc}
\hline PREVENCIÓN CONTRA INCENDIOS & SI & NO & POND. \\
\hline ¿Existe identificación clara a los medios para combatir y extinguir el incendio? & $\checkmark$ & & 4 \\
¿Existe fácil acceso a los medios para combatir y extinguir el incendio? & $\checkmark$ & 3 \\
¿Cuentan con procedimientos de evacuación y control de incendios? & & $\checkmark$ & 0 \\
¿Se cuenta con extintores? & & $\checkmark$ & 3 \\
¿Se cuenta con un sistema de extinción de fuego mediante uso de agua? & & $\checkmark$ & 0 \\
¿Se cuenta con alarma para avisar siniestros? & & 1,67 \\
Ponderación del área: & SI & NO & POND. \\
\hline HERRAMIENTAS Y MÁQUINAS & $\checkmark$ & & 3 \\
\hline ¿Se les proporciona un mantenimiento adecuado? & $\checkmark$ & 4 \\
¿Se utilizan de manera adecuada? & $\checkmark$ & 4 \\
¿Se le proporciona entrenamiento adecuado al personal para su uso? & & $\checkmark$ & 3 \\
¿Se siguen procedimientos de uso de máquina? & & 3,5 \\
Ponderación del área: & SI & NO & POND. \\
\hline AMBIENTE LABORAL & $\checkmark$ & & 5 \\
\hline ¿Es cordial? & & $\checkmark$ & 4 \\
¿Existen distracciones para el personal en las áreas de trabajo? & $\checkmark$ & 4 \\
¿Los operarios juegan bromas o hacen sorpresas? & $\checkmark$ & 5 \\
¿Existe comunicación entre operarios y el jefe inmediato superior? & & 4,5 \\
Ponderación del área: & & & \\
\hline
\end{tabular}

Ponderación: Excelente (5), Muy Bueno (4), Bueno (3), Regular (2), Malo (1), Muy Malo (0)

\section{Seguridad Industrial: Guía de auditoria}

Condiciones actuales.

Departamento: Almacén General.

Fecha de inspección: 5 de agosto del 2013.

Auditor. SCJJ

\begin{tabular}{lccc}
\hline MANEJO DE MATERIALES & SI & NO & POND. \\
\hline ¿Tiene base sólida? & & $\checkmark$ & 3 \\
¿Se opera con seguridad la máquina para estibar? & $\checkmark$ & & 4 \\
¿Se utiliza carga adecuada a la capacidad de montacarga? & $\checkmark$ & & 4 \\
¿Está el personal entrenado para el manejo de materiales? & & $\checkmark$ & 3 \\
¿Existen procedimientos para el manejo de materiales? & & $\checkmark$ & 2 \\
¿Se utilizan las precauciones debidas cuando hay riesgo? & $\checkmark$ & & 3 \\
¿Se utiliza equipo de protección personal? & & & 4 \\
¿Están plenamente identificados los materiales que se manipulan? & & & 3,25 \\
Ponderación del área: & & &
\end{tabular}




\begin{tabular}{|c|c|c|c|}
\hline PASILLOS E INSTALACIONES & SI & NO & POND. \\
\hline ¿Los atraviesan mangueras o cables eléctricos? & $\checkmark$ & & 3 \\
\hline ¿Se encuentran plenamente identificados? & $\checkmark$ & & 4 \\
\hline $\begin{array}{l}\text { ¿Se cumplen normas de orden de limpieza de forma que no dificulte la } \\
\text { circulación en los pasillos? }\end{array}$ & $\checkmark$ & & 4 \\
\hline $\begin{array}{l}\text { ¿Se cuenta con la debida señalización cromática en las diferentes áreas } \\
\text { de trabajo? }\end{array}$ & $\checkmark$ & & 4 \\
\hline ¿Son adecuadas las condiciones de iluminación en el área? & & $\checkmark$ & 3 \\
\hline ¿Son adecuadas las condiciones de temperatura y ventilación en el área? & & $\checkmark$ & 2 \\
\hline ¿Existe mucho ruido en el área de trabajo? & & $\checkmark$ & 3 \\
\hline ¿Existe mucha vibración en el área de trabajo? & & $\checkmark$ & 3 \\
\hline $\begin{array}{l}\text { ¿Se encuentra plenamente identificado el equipo de primeros auxilios } \\
\text { en las áreas? }\end{array}$ & & $\checkmark$ & 0 \\
\hline $\begin{array}{l}\text { ¿Se encuentran en buenas condiciones las paredes y techos de las } \\
\text { instalaciones? }\end{array}$ & & $\checkmark$ & 3 \\
\hline ¿El piso instalado en cada área es el adecuado según las tareas que se realizan? & $\checkmark$ & & 4 \\
\hline $\begin{array}{l}\text { ¿Se encuentra en buenas condiciones el sistema de cableado eléctrico } \\
\text { de la empresa? }\end{array}$ & & $\checkmark$ & 3 \\
\hline ¿Se localiza algún material combustible cerca de la fuente de energía? & $\checkmark$ & & 0 \\
\hline Ponderación del área: & & & 2,77 \\
\hline
\end{tabular}

\begin{tabular}{lccc}
\hline HERRAMIENTAS Y MÁQUINAS & SI & NO & POND. \\
\hline ¿Se guardan en un lugar adecuado las herramientas cuando no están en uso? & $\checkmark$ & & 5 \\
¿La maquinaria cuenta con un equipo de protección? & $\checkmark$ & & 4 \\
¿Se les proporciona un mantenimiento adecuado? & & $\checkmark$ & 2 \\
¿Se utilizan de manera adecuada? & & & 4 \\
¿Se le proporciona entrenamiento adecuado al personal para su uso? & $\checkmark$ & & 3 \\
¿Maniobran de forma segura las diferentes máquinas de trabajo? & $\checkmark$ & 4 \\
¿Se siguen procedimientos de uso de maquina? & & $\checkmark$ & 2 \\
¿Se toman medidas precautorias para dar mantenimiento a la maquinaria? & $\checkmark$ & 4 \\
¿Se utiliza aisladores de sonido en las máquinas de producción? & & 3,56 \\
Ponderación del área: & &
\end{tabular}

\begin{tabular}{lccc}
\hline EQUIPO DE PROTECCIÓN PERSONAL & SI & NO & POND. \\
\hline ¿Se utiliza equipo de protección auditiva? & $\checkmark$ & 3 \\
¿Se utiliza equipo de protección respiratoria? & $\checkmark$ & 3 \\
¿Se utiliza ropa de protección de cuerpo? & & $\checkmark$ & 0 \\
¿Se utilizan protectores de manos y extremidades superiores? & $\checkmark$ & 5 \\
¿Se utiliza calzado de protección y protectores de extremidades inferiores? & & $\checkmark$ & 2 \\
Ponderación del área: & & 2,6 \\
\hline
\end{tabular}




\begin{tabular}{lccc}
\hline AMBIENTE LABORAL & SI & NO & POND. \\
\hline ¿ES cordial? & $\checkmark$ & & 3 \\
¿Existen distracciones para el personal en las áreas de trabajo? & & $\checkmark$ & 4 \\
¿Los operarios juegan bromas o hacen sorpresas? & & $\checkmark$ & 4 \\
¿Existe comunicación entre operarios y el jefe inmediato superior? & & & 4 \\
Ponderación del área: & & 2,6
\end{tabular}

Ponderación: Excelente (5), Muy Bueno (4), Bueno (3), Regular (2), Malo (1), Muy Malo (0)

Seguridad Industrial: Guía de auditoría

Condiciones actuales.

Departamento: Producción.

Fecha de inspección: 5 de agosto del 2013.

Auditor. SZLR

\begin{tabular}{lccc}
\hline MANEJO DE MATERIALES & SI & NO & POND. \\
\hline ¿Tiene base sólida? & $\checkmark$ & & 3 \\
¿Se opera con seguridad la máquina para estibar? & $\checkmark$ & 4 \\
¿Se utiliza carga adecuada a la capacidad de montacarga? & $\checkmark$ & 3 \\
¿Está el personal entrenado para el manejo de materiales? & $\checkmark$ & & 3 \\
¿Existen procedimientos para el manejo de materiales? & & $\checkmark$ & 2 \\
¿Se utilizan las precauciones debidas cuando hay riesgo? & $\checkmark$ & & 3 \\
¿Se utiliza equipo de protección personal? & & $\checkmark$ & 4 \\
¿Están plenamente identificados los materiales que se manipulan? & & & 3,0 \\
Ponderación del área: & & &
\end{tabular}

\begin{tabular}{lccc}
\hline PASILLOS E INSTALACIONES & SI & NO & POND. \\
\hline ¿LOS atraviesan mangueras o cables eléctricos? & $\checkmark$ & & 2 \\
¿Se encuentran plenamente identificados? & $\checkmark$ & 3 \\
¿Se cumplen normas de orden de limpieza de forma que no dificulte la & & & \\
circulación en los pasillos? & $\checkmark$ & & 3 \\
¿Se cuenta con la debida señalización cromática en las diferentes áreas de trabajo? & $\checkmark$ & & 3 \\
¿Son adecuadas las condiciones de iluminación en el área? & $\checkmark$ & 5 \\
¿Son adecuadas las condiciones de temperatura y ventilación en el área? & & $\checkmark$ & 3 \\
¿Existe mucho ruido en el área de trabajo? & $\checkmark$ & 2 \\
¿Existe mucha vibración en el área de trabajo? & & $\checkmark$ & 2 \\
¿Se encuentran plenamente identificados el equipo de primeros auxilios en las áreas? & & $\checkmark$ & 2 \\
¿Se encuentran en buenas condiciones las paredes y techos de las instalaciones? & $\checkmark$ & & 3 \\
¿El piso instalado en cada área es el adecuado según las tareas que se realizan? & $\checkmark$ & & 4 \\
¿Se encuentra en buenas condiciones el sistema de cableado eléctrico de la empresa? & $\checkmark$ & 2 \\
¿Se localiza algún material combustible cerca de la fuente de energía? & $\checkmark$ & & 2 \\
Ponderación del área: & & & 2,77 \\
\hline
\end{tabular}




\begin{tabular}{lccc}
\hline PREVENCIÓN CONTRA INCENDIOS & SI & NO & POND. \\
\hline ¿Existe identificación clara a los medios para combatir y extinguir el incendio? & $\checkmark$ & & 4 \\
¿Existe fácil acceso a los medios para combatir y extinguir el incendio? & $\checkmark$ & 4 \\
¿Cuentan con procedimientos de evacuación y control de incendios? & & $\checkmark$ & 2 \\
¿Se cuenta con extintores? & $\checkmark$ & 4 \\
¿Se cuenta con un sistema de extinción de fuego mediante uso de agua? & $\checkmark$ & 4 \\
¿Se utiliza equipo de protección personal? & $\checkmark$ & 3 \\
¿Se cuenta con alarma para avisar siniestros? & $\checkmark$ & 5 \\
Ponderación del área: & & 3,71
\end{tabular}

HERRAMIENTAS Y MÁQUINAS

¿Se guardan en un lugar adecuado las herramientas cuando no están en uso?

¿La maquinaria cuenta con un equipo de protección?

¿Se les proporciona un mantenimiento adecuado?

¿Se utilizan de manera adecuada?

¿Se le proporciona entrenamiento adecuado al personal para su uso?

¿Maniobran de forma segura las diferentes máquinas de trabajo?

¿Se siguen procedimientos de uso de maquina?

¿Se toman medidas precautorias para dar mantenimiento a la maquinaria?

¿Se utiliza aisladores de sonido en las máquinas de producción?

Ponderación del área:

\begin{tabular}{|c|c|c|c|}
\hline EQUIPO DE PROTECCIÓN PERSONAL & SI & NO & POND. \\
\hline ¿Se utiliza equipo de protección auditiva? & $\checkmark$ & & 4 \\
\hline ¿Se utiliza equipo de protección respiratoria? & $\checkmark$ & & 4 \\
\hline ¿Se utiliza ropa de protección de cuerpo? & $\checkmark$ & & 3 \\
\hline ¿Se utiliza protectores de manos y extremidades superiores? & $\checkmark$ & & 4 \\
\hline ¿Se utiliza calzado de protección y protectores de extremidades inferiores? & $\checkmark$ & & 4 \\
\hline
\end{tabular}

Ponderación del área:

\begin{tabular}{lccc}
\hline AMBIENTE LABORAL & SI & NO & POND. \\
\hline ¿Es cordial? & $\checkmark$ & & 3 \\
¿Existen distracciones para el personal en las áreas de trabajo? & & $\checkmark$ & 4 \\
¿Los operarios juegan bromas o hacen sorpresas? & $\checkmark$ & & 3 \\
¿Existe comunicación entre operarios y el jefe inmediato superior? & & $\checkmark$ & 2 \\
Ponderación del área: & & 3,0
\end{tabular}

Ponderación: Excelente (5), Muy Bueno (4), Bueno (3), Regular (2), Malo (1), Muy Malo (0) 


\section{DISCUSIÓN}

CONDICIONES ACTUALES DEL DEPARTAMENTO DE ADMINISTRACIÓN

\begin{tabular}{|c|c|}
\hline $\begin{array}{l}\text { AREA DE } \\
\text { ANÁLISIS }\end{array}$ & PROBLEMÁTICAS \\
\hline $\begin{array}{l}\text { Pasillos e } \\
\text { instalaciones }\end{array}$ & $\begin{array}{l}\text { - Es necesaria la identificación de áreas de circulación y rutas de } \\
\text { acceso y evacuación, para los cuales debe considerarse procedi- } \\
\text { mientos respectivos. } \\
\text { - Se deben señalar las salidas de emergencia para evitar reacciones y } \\
\text { desarrollar contingencias derivadas de algún accidente o siniestro. } \\
\text { - Es necesario que se tenga una buena ubicación de los equipos de } \\
\text { primeros auxilios. } \\
\text { - Se debe tener más cuidado con los cables de instalación eléctrica. } \\
\text { Una mala ubicación de estos puede ser causa de un siniestro. } \\
\text { - Las condiciones de limpieza se consideran óptimas. }\end{array}$ \\
\hline $\begin{array}{l}\text { Prevención } \\
\text { contra } \\
\text { incendios }\end{array}$ & $\begin{array}{l}\text { - Se debe retirar todos los elementos que dificultan el rápido acceso a } \\
\text { los medios para extinción de incendios. } \\
\text { - Es necesario la creación e implementación de un procedimiento de } \\
\text { evacuación en caso de siniestros o incendios. } \\
\text { - Es necesario la tenencia de más de un extintor en el área de adminis- } \\
\text { tración, así como extintores de agua. } \\
\text { - Es necesaria la implementación de una alarma en caso de incendio } \\
\text { en el área evaluada. }\end{array}$ \\
\hline $\begin{array}{l}\text { Herramientas } \\
\text { y máquinas }\end{array}$ & $\begin{array}{l}\text { - Se debe prever el mantenimiento de los equipos que utiliza el área } \\
\text { evaluada, ya sea de manera trimestral o semestral. }\end{array}$ \\
\hline $\begin{array}{l}\text { Ambiente } \\
\text { laboral }\end{array}$ & $\begin{array}{l}\text { - La relación entre los trabajadores es muy amena y se percibe cor- } \\
\text { dialidad y confianza entre ello. } \\
\text { - Existe un nivel alto de comunicación entre todo el personal de esta } \\
\text { área. }\end{array}$ \\
\hline
\end{tabular}


CONDICIONES ACTUALES DEL DEPARTAMENTO DE ALMACEN GENERAL

\begin{tabular}{|c|c|}
\hline $\begin{array}{l}\text { AREA DE } \\
\text { ANÁLISIS }\end{array}$ & PROBLEMÁTICAS \\
\hline $\begin{array}{l}\text { Manejo de } \\
\text { materiales }\end{array}$ & $\begin{array}{l}\text { - Se determinó en esta área un alto grado de efectividad en el manejo } \\
\text { de materias primas, de manera eficiente y segura el equipo para su } \\
\text { transporte. } \\
\text { - A pesar de no contar con normas o procedimientos que establezcan } \\
\text { su ubicación y manejo, las materias primas están bien organizadas. } \\
\text { - Las condiciones del equipo de manejo de materiales son acepta- } \\
\text { bles, considerando que no se le proporciona un mantenimiento } \\
\text { adecuado, así como la inexistencia de normas y procedimientos que } \\
\text { reflejen el buen estado de la maquinaria. }\end{array}$ \\
\hline $\begin{array}{l}\text { Pasillos e } \\
\text { instalaciones }\end{array}$ & $\begin{array}{l}\text { - Esta área presenta algunos inconvenientes en materia de seguridad, } \\
\text { - Lue deberá considerarse para la prevención de algún accidente. } \\
\text { de evacuación, medios de prevención contra incendios o señalización } \\
\text { de áreas delimitadas especiales están debidamente identificadas. } \\
\text { - El entorno de las instalaciones se considera adecuados para las tareas } \\
\text { que se realizan dentro de esta área. El piso es reforzado; existen muy } \\
\text { buenas condiciones de iluminación, temperatura y ventilación. } \\
\text { - Existen altos riesgos de siniestros debido a la existencia de materia } \\
\text { combustible cerca de fuentes de energía dentro de la planta, como } \\
\text { son los circuitos eléctricos expuestos. } \\
\text { - Respecto a la limpieza dentro de la planta, mantiene parámetros } \\
\text { aceptables. } \\
\text { - Un aspecto importante a considerar es la renovación del área donde } \\
\text { se ubica el producto terminado, ya que comparte el área con el labo- } \\
\text { ratorio de químicos para el pintado del mismo. }\end{array}$ \\
\hline $\begin{array}{l}\text { Prevención } \\
\text { contra } \\
\text { incendios }\end{array}$ & $\begin{array}{l}\text { - Se identificó la existencia de medios preventivos en situación muy } \\
\text { limitada, ya que la distribución de extintores no es la adecuada. } \\
\text { - No existe ningún procedimiento que dicte medidas a seguir frente a } \\
\text { un siniestro. } \\
\text { - El equipo de protección no es el adecuado de los electricistas; no } \\
\text { cuentan con medios suficientes para todo el personal involucrado. }\end{array}$ \\
\hline
\end{tabular}




\begin{tabular}{|c|c|}
\hline $\begin{array}{l}\text { AREA DE } \\
\text { ANÁLISIS }\end{array}$ & PROBLEMÁTICAS \\
\hline $\begin{array}{l}\text { Herramientas } \\
\text { y máquinas }\end{array}$ & $\begin{array}{l}\text { - Esta área cuenta con un ambiente especial para el almacenamiento } \\
\text { del equipo de manejo de materiales, aunque es preferible aislarlo } \\
\text { con el fin de garantizar el buen estado de la maquinaria y establecer } \\
\text { restricción de acceso a esta. } \\
\text { - No existen procedimientos para el uso de la maquinaria, la capacita- } \\
\text { ción de los nuevos empleados es mínima y sin base científica. } \\
\text { - No se toman en cuenta medidas de seguridad para manejo de equipo } \\
\text { como uso de mecanismos de protección de la maquina en determina- } \\
\text { das situaciones: potencia en los botales para el lavado del cuero. } \\
\text { - Respecto al mantenimiento se estableció que no existe algún proce- } \\
\text { so de control de la maquinaria, por lo que se incurre en gastos bajo } \\
\text { concepto de mantenimiento correctivo. Esto puede representar } \\
\text { atrasos en las operaciones de esta área y la continuación del proce- } \\
\text { so productivo. }\end{array}$ \\
\hline $\begin{array}{l}\text { Ambiente } \\
\text { laboral }\end{array}$ & $\begin{array}{l}\text { - El ambiente laboral es muy ameno, lo que refleja buen grado de } \\
\text { comunicación de información. } \\
\text { - Se recomienda concientizar al personal en cuanto a que las bromas } \\
\text { y distractores representan generadores de accidentes en el área de } \\
\text { trabajo. }\end{array}$ \\
\hline
\end{tabular}

\section{CONDICIONES ACTUALES DEL DEPARTAMENTO DE PRODUCCIÓN}

\begin{tabular}{|c|c|}
\hline $\begin{array}{c}\text { AREA DE } \\
\text { ANÁLISIS }\end{array}$ & \multicolumn{1}{c|}{ PROBLEMÁTICAS } \\
\hline $\begin{array}{l}\text { Manejo de } \\
\text { materiales }\end{array}$ & $\begin{array}{l}\text { - Se debe tener un mejor cuidado con el tratamiento de insumos quí- } \\
\text { micos, ya que por un mal uso pueden ocurrir siniestros. } \\
\text { - Debe existir un manual de procedimientos en el uso de los insumos. } \\
\text { - Deben realizarse capacitaciones al personal que se va incorporando } \\
\end{array}$ \\
$\begin{array}{l}\text { a la producción en la empresa. } \\
\text { - Los materiales que se utilizan deben estar ubicados en su respectivo } \\
\text { lugar; asimismo deben ser fácil de identificarse. }\end{array}$ \\
\hline
\end{tabular}




\begin{tabular}{|c|c|}
\hline $\begin{array}{l}\text { AREA DE } \\
\text { ANÁLISIS }\end{array}$ & PROBLEMÁTICAS \\
\hline $\begin{array}{l}\text { Pasillos e } \\
\text { instalaciones }\end{array}$ & $\begin{array}{l}\text { - Este es el departamento de mayor movimiento de materiales y per- } \\
\text { sonal. Se considera importante establecer los respectivos pasillos } \\
\text { de circulación, para que no se entorpezca el proceso y se reduzcan } \\
\text { los riesgos mencionados en la sección anterior. } \\
\text { - Al establecer las áreas para la circulación de personal se ayudará a } \\
\text { identificar las diferentes áreas de este departamento; asimismo se } \\
\text { facilitara la identificación de rutas de acceso y evacuación. } \\
\text { - Se debe considerar la señalización de la disposición de la } \\
\text { maquinaria utilizada en el proceso, de la misma manera la debida } \\
\text { identificación de los medios de prevención contra incendios, } \\
\text { primeros auxilios o señalización de áreas delimitadas especiales } \\
\text { como depósito de desechos o residuos. } \\
\text { - Existen altos riesgos de siniestro debido a la existencia de materia } \\
\text { combustible cerca de las fuentes de ignición como son circuitos } \\
\text { eléctricos expuestos que se identificaron. } \\
\text { - Se estableció que periódicamente se realiza limpieza del área de } \\
\text { producción debido a la gran cantidad de desecho derivada del } \\
\text { proceso productivo. }\end{array}$ \\
\hline $\begin{array}{l}\text { Prevención } \\
\text { contra } \\
\text { incendios }\end{array}$ & $\begin{array}{l}\text { - En la industria de cuero es de vital importancia contar con los } \\
\text { medios y planes de contingencias para contrarrestar incendios en } \\
\text { toda elárea operativa. } \\
\text { - Se identificó la falta de medios adecuados para hacer frente a un } \\
\text { incendio y la inexistencia de medios preventivos. } \\
\text { - No se cuenta con normas y procedimientos para el control, } \\
\text { prevención o evacuación. Ello representa un alto riesgo para las } \\
\text { operaciones de la empresa, ya que de no poder controlar un } \\
\text { pequeño siniestro producido se pondría en peligro, no solo la } \\
\text { integridad de los trabajadores, sino de la maquinaria; ambos forman } \\
\text { parte importante en el proceso de producción de la empresa objeto } \\
\text { de estudio. }\end{array}$ \\
\hline
\end{tabular}




\begin{tabular}{|c|c|}
\hline $\begin{array}{l}\text { AREA DE } \\
\text { ANÁLISIS }\end{array}$ & PROBLEMÁTICAS \\
\hline $\begin{array}{l}\text { Equipo de } \\
\text { protección } \\
\text { personal }\end{array}$ & $\begin{array}{l}\text { - En relación con la encuesta realizada en los diferentes sectores de la } \\
\text { empresa, el área de producción registra el mayor número de acci- } \\
\text { dentes. } \\
\text { - El equipo que se proporciona al trabajador consta de lentes de pro- } \\
\text { tección, guantes, mascarillas, etc. } \\
\text { - Se determinó la necesidad de hacer conciencia del uso del equipo de } \\
\text { protección por parte del personal, el cual de acuerdo a la encuesta } \\
\text { realizada, no lo utiliza por considerar molesto su uso. }\end{array}$ \\
\hline $\begin{array}{l}\text { Ambiente } \\
\text { laboral }\end{array}$ & $\begin{array}{l}\text { - Existe cierto de grado de cordialidad entre los trabajadores. } \\
\text { - Se debe incentivar la comunicación más seguida sobre la informa- } \\
\text { empleados u operarios. } \\
\text { - Algunos operarios tienden a hacer bromas a compañeros de trabajo. } \\
\text { Esto representa un peligro de accidente latente, puesto que deriva } \\
\text { posibles actos inseguros por parte de los trabajadores objeto de las } \\
\text { bromas. }\end{array}$ \\
\hline
\end{tabular}

\section{CONCLUSIONES}

$1^{\mathrm{a}}$. Los resultados de reducción de vulnerabilidades correspondientes a accidentes y siniestros reflejarán mejoras en materia de productividad, autocontrol y aplicación de planes de contingencia, producto de inspecciones programáticas encaminadas a velar por la integridad física del recurso humano.

$2^{\mathrm{a}}$. En la actualidad, las empresas nacionales, como la empresa objeto de estudio, se enfrentarán a un mundo globalizado, el cual viene a imponer la necesidad de tomar medidas respectivas para ser más competitivos. Dentro de estas medidas está la creación de un programa de seguridad industrial, el cual garantizará en gran medida un buen desempeño de las labores productivas de los diferentes sectores del país.

$3^{\mathrm{a}}$. La implementación de un programa de seguridad industrial es de vital importancia, ya que con ello se establecerá las condiciones óptimas de fun- 
cionamiento para reducir sustancialmente los parámetros estadísticos de accidentes laborales y siniestros; asimismo se reducirá el nivel de improductividad que se originaria por estas causas.

$4^{\mathrm{a}}$. Se deben implementar medidas de seguridad que garanticen el bienestar de todos los individuos de las organizaciones empresariales; debido a lo anterior, no dificultará los fines de cada uno de los componentes de una empresa, empleador y trabajador para que se alcancen sus objetivos con mayor eficiencia y eficacia si se proveen los medios adecuados que permitan reducir contratiempos o desembolsos monetarios derivados de accidentes laborales.

$5^{\mathrm{a}}$. Un programa de seguridad se considera como un instrumento de gestión dentro de una organización, que si bien en un inicio cuantitativamente no se puede determinar su incidencia en la productividad, cualitativamente inicia su incidencia dentro de la gestión de la empresa, afectando posteriormente al aspecto cuantitativo en gran manera.

\section{REFERENCIAS BIBLIOGRÁFICAS}

BERNAL, C. (2000). Metodología de la investigación: para Administración y Economía. Bogotá: Pearson Educación.

BRANDENSTEIN, B. (1983). Cuestiones fundamentales de la filosofía. Barcelona Herder.

Bunge, M. (1972). La investigación científica: su estrategia y su filosofía. Barcelona: Ariel.

Ceballos, R. (2009). Prevención de riesgos laborales para administrativo, auxiliar administrativo y técnicos.

COOPERS \& LYBRAND (2010). Manual de auditoría interna. Control interno, auditoria y seguridad.

FAGOTHELY, A. (1973). Ética: teoría y aplicación. México D.F.: Mcgraw-Hill.

GARCIA, D. (2004). Ética empresarial: del diálogo a la confianza. Madrid: Trotta.

HARRINSON L. (2011). Manual de auditoría medioambiental, higiene y seguridad.

HumE, D. (2009). Investigación sobre el entendimiento humano.

Kataya, R. (2003). Introducción a la filosofía. Lima, Universidad Ricardo Palma. Editorial Universitaria.

SCHMidT, E. (2000). Ética y negocios para América Latina. Lima. Biblioteca Universitaria. Universidad del Pacífico. 